\title{
Origin of Slow Solvation Dynamics in DNA: DAPI in Minor Groove of Dickerson-Drew DNA
}

\author{
Deepika Sardana, ${ }^{\star}$ Kavita Yadav, ${ }^{\star}$ Him Shweta, Ndege Simisi Clovis, Parvez Alam and Sobhan Sen*
}

Spectroscopy Laboratory, School of Physical Sciences, Jawaharlal Nehru University, New Delhi 110067, India

*E-mail: sens@mail.jnu.ac.in

Matching and Stokes Shift Data Reproducibility: Solvation dynamics in DNA continue over broad time-range. Hence, a single experimental technique does not cover the entire time-range of dynamics. We combined TRES from
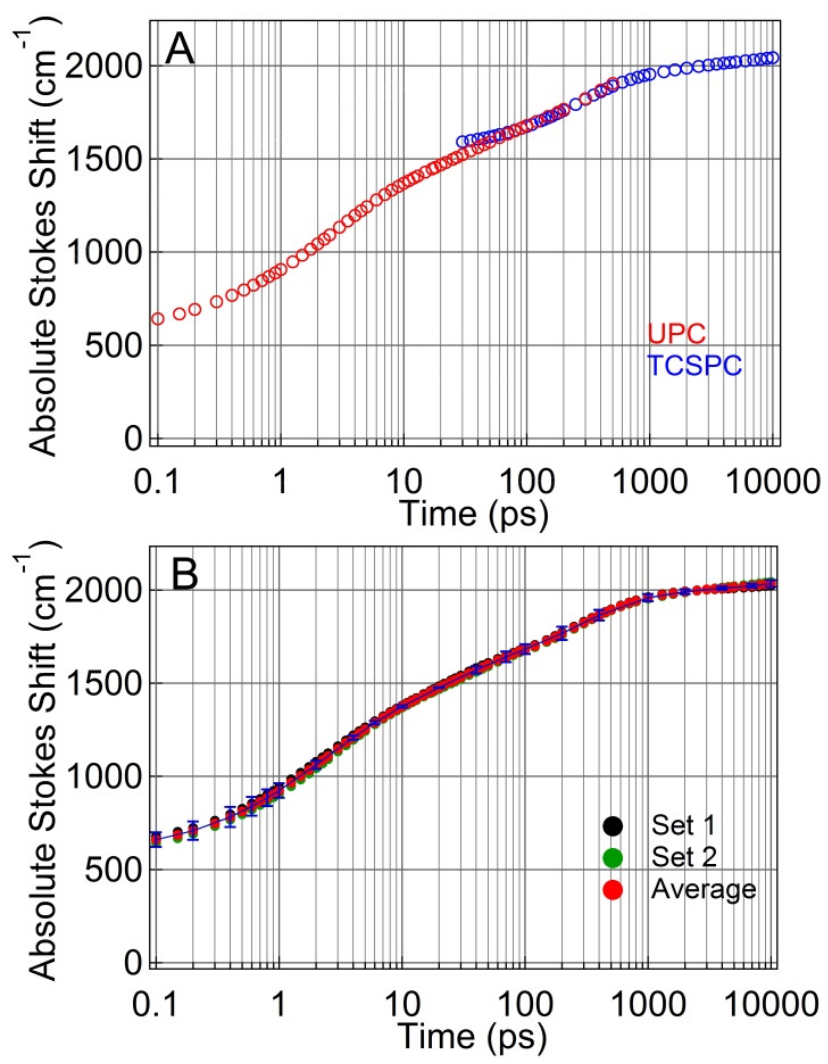

Figure S1. (A) The matching of absolute Stokes shift data of DAPI in Dickerson-DNA from UPC (red) and TCSPC (blue) data. Matching of data is excellent at common time-points from $\sim 50 \mathrm{ps}$ to 500 ps. (B) Reproducibility of Stokes shift data obtained from two independent sets of measurements performed with a gap of nearly four years. Plot also includes the average of the two datasets, which is presented in the manuscript. Errors bars $( \pm$ SD) were calculated after including several other Stokes shift data-sets of other DAPI/duplex-DNA complexes reported earlier.
UPC and TCSPC data which allowed us to cover a broad time-range of five decades from 100 fs to $10 \mathrm{~ns}$. We measured UPC and TCSPC data in such a manner that some range of common time-points exist in the final Stokes shift data. The constructed absolute Stokes shifts from two techniques were obtained as $1^{\text {st }}$ moment frequency shift. Figure S1A shows the matching of Stokes shift data at the common time-points obtained from UPC and TCSPC. The data of UPC and TCSPC match very well at common timepoints from $\sim 50 \mathrm{ps}$ to $\sim 500 \mathrm{ps}$.

Two independent sets of UPC and TCSPC data on separate DAPI/Dickerson-Drew DNA samples were collected, and subsequently, two sets of absolute Stokes shifts were calculated. (Note that these two data-sets were measured with a gap of nearly four years and with different concentrations of DAPI and DNA.) Figure S1B shows the two sets of absolute Stokes shifts data along with their average. The two data-sets are very similar, showing dispersed solvation dynamics in DNA which continue over five decades of time. The average absolute Stokes shift data is presented in Figure 4B of main manuscript. 


\section{SIMULATION DATA}

Table S1. Excited state atomic charges for DAPI generated through two-stage restrained electrostatic potential (RESP) charge methodology, subsequent to generation of electrostatic potential of DAPI at CIS level (see Methods).

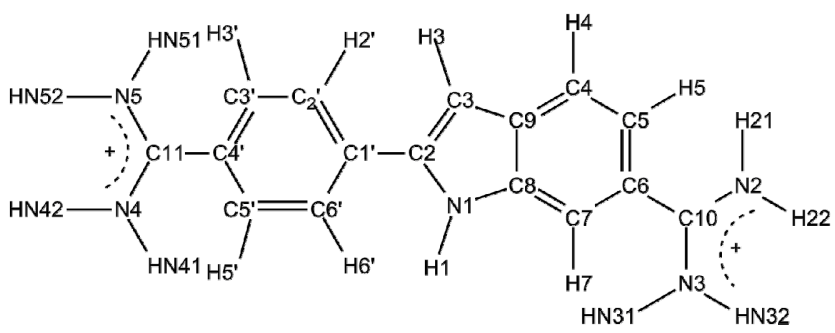

\begin{tabular}{|c|c|c|c|}
\hline Atom Name & Charge (a.u.) & Atom Name & Charge (a.u.) \\
\hline N1 & -0.355989 & H1 & 0.361424 \\
\hline C2 & 0.095217 & N4 & -0.658260 \\
\hline C3 & -0.156048 & N5 & -0.658260 \\
\hline C5 & -0.213669 & H3 & 0.191185 \\
\hline C6 & -0.134840 & H4 & 0.202224 \\
\hline C7 8 & -0.014997 & H5 & 0.156363 \\
\hline C9 & -0.245286 & H2' & 0.170887 \\
\hline C10 & 0.175048 & H3' & 0.1702518 \\
\hline N2 & 0.118057 & H5' & 0.169969 \\
\hline N3 $1 '$ & 0.474885 & H6' & 0.142456 \\
\hline C2 & -0.658260 & H21 & 0.399521 \\
\hline C3' & -0.658260 & H22 & 0.399521 \\
\hline C4' & 0.065350 & HN31 & 0.399521 \\
\hline C5' & -0.134036 & HN32 & 0.399521 \\
\hline C6' 11 & -0.159581 & HN41 & 0.399521 \\
\hline & 0.001738 & HN42 & 0.399521 \\
\hline
\end{tabular}



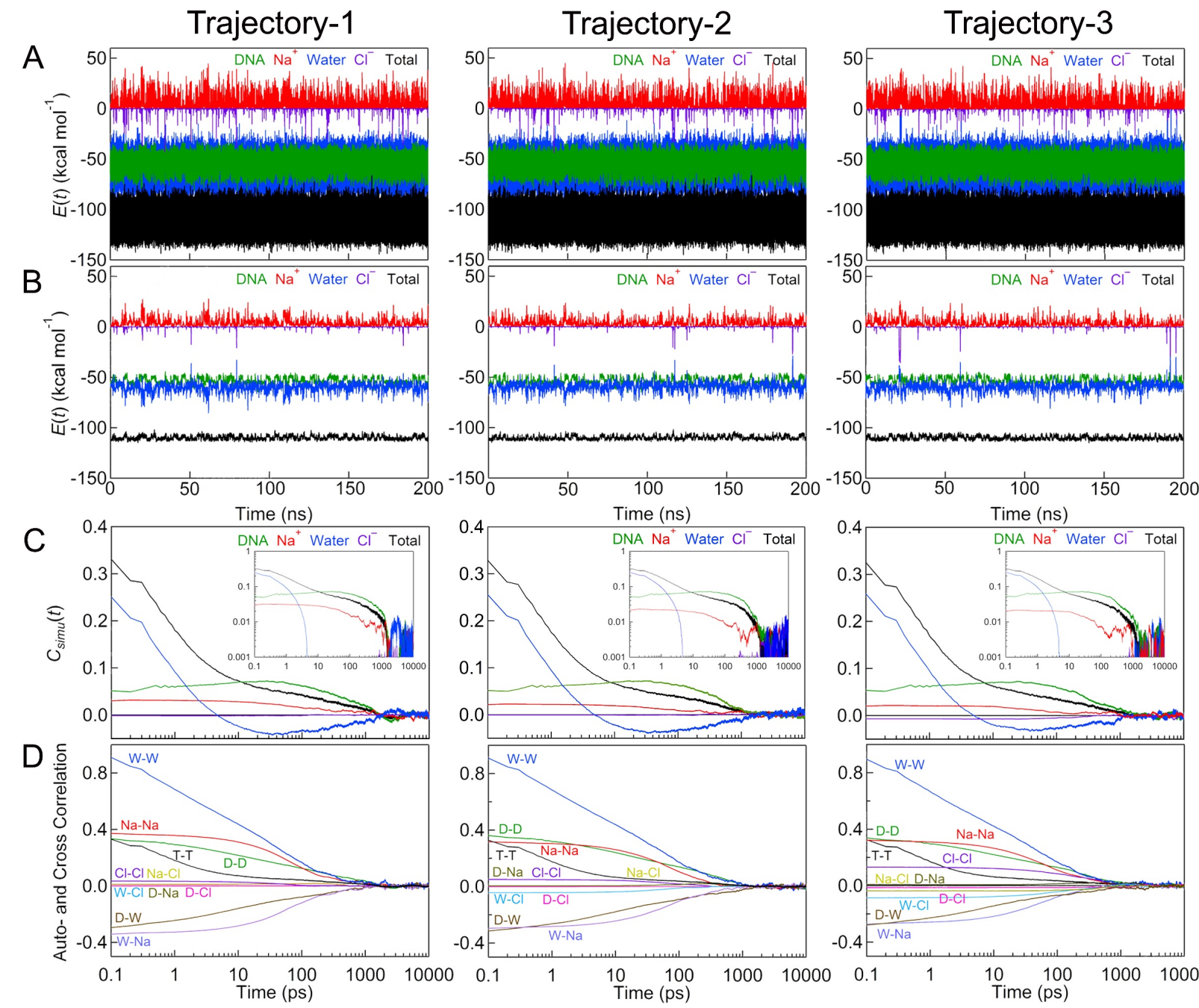

Trajectory-3

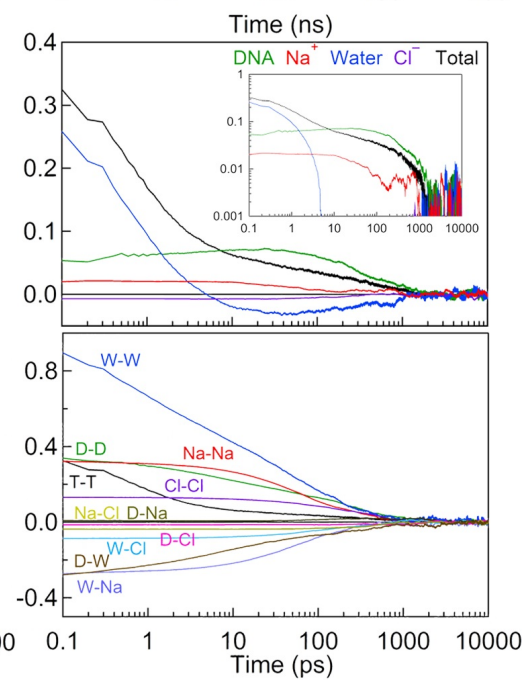

Figure S2. Panels in (A) show the raw electrostatic interaction energy fluctuations of excited-state DAPI with $\mathrm{Na}^{+}$(red), $\mathrm{Cl}^{-}$(purple), water (blue), DNA (green) and total system (black) over $200 \mathrm{~ns}$ simulation calculated using DSF sum method (equation-1 in main manuscript) from the three independent trajectories. Panels in (B) show the same energy fluctuations after smoothing with 100 ps running average which clearly show the fluctuations and the anti-correlations among components. Panels in (C) plot the total TCF and the partial-TCFs of individual components calculated using equation-4 of main manuscript. Insets show the same data in log-log plot. Panels in (D) show the auto- and cross-correlations of the individual components as well as the total correlations, calculated using equation-5 from three trajectories. The data in panels (C) and (D) show good reproducibility, which are obtained from the three trajectories. Average of data in panel $(\mathrm{C})$ is reported in the main manuscript.

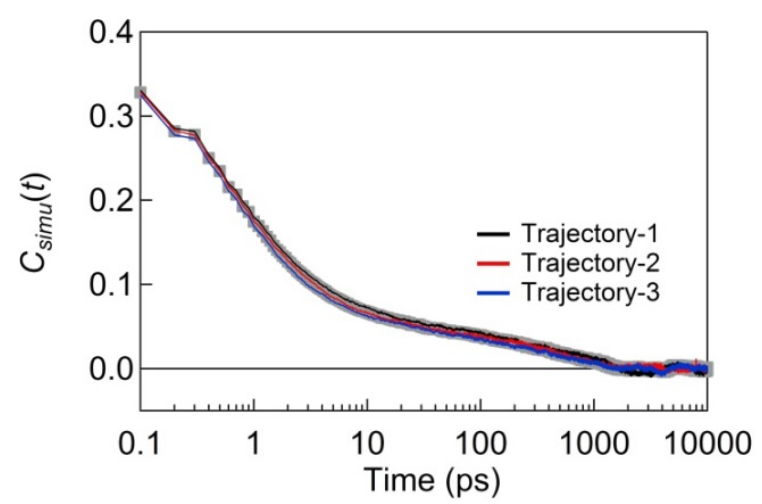

Figure S3. Reproducibility of total TCFs obtained from three independent (200 ns) trajectories. The average of the three TCFs is reported in main manuscript. The error $( \pm \mathrm{SD})$ is shown as grey shaded bars. 